\title{
$\underline{\mathbf{P}-175}$
}

\section{Molecular Insights on Oligostilbenoid Biosynthesis}

\author{
Bayach I ${ }^{\mathrm{a}, \mathrm{b}}$, Sancho Garcia J.C ${ }^{\mathrm{c}}$, Di Meo F $F^{\mathrm{a}}$, Nurhuda $\mathrm{M}^{\mathrm{b}}$, Weber J.F.F $\mathrm{F}^{\mathrm{b}}$ and Trouillas $\mathrm{P}^{\mathrm{a}, \mathrm{d}, *}$ \\ ${ }^{a}$ LCSN - EA1069, Faculté de Pharmacie, 2 rue du Docteur Marcland, Limoges, France; ${ }^{b}$ Atta-ur-Rahman Institute for \\ Natural Product Discovery (RiND) Faculty of Pharmacy, Universiti Teknologi MARA, 42300, Bandar Puncak \\ Alam,Selangor, Malaysia; 'Departamento Química Física, Universidad de Alicante, Ap. De Correos 99, E-03080, \\ Alicante, Spain; ${ }^{d}$ Service de Chimie des Matériaux Nouveaux, Université de Mons-Hainaut, Mons, Belgique; \\ E-mail: patrick.trouillas@unilim.fr
}

Stilbene dimerisation with help of oxidants is a reaction used to biomimetically synthesise oligostilbenes, a small group of polyphenols abundantly present in dipterocarps, a major timber resource in different parts of the world including Malaysia. The fascination for these compounds originates from the huge variety in their chemical structures. From the literature, however, the oligomerisation reaction is poorly understood. A comprehensive mechanistic theory on the determinism of the stilbene dimerisation by metal oxidants was recently proposed. Even if providing a remarkable predictive approach, a full theoretical backup is still to be established. This work aims at using DFT to validate this theory. The parameters thought to play an important role in this synthesis (e.g., ionisation potential, non-covalent interaction including $\pi$-stacking, solvent effects, metal complexation) will be studied. These parameters will allow to fully rationalizing the stilbenoid biosynthesis process step by step. A particular attention is given to the formation of pre-reaction $\pi$-stacking complexes between two dimers (Figure 1) i.e., prior to the regio- and stereo-selective formation of tetramers. The use of the adequate DFT-D methodology appears mandatory to properly describe the dispersive interactions existing between both dimers.

Keywords: Oligostilbenes, Biosynthesis, $\pi$-stacking complexation. 